\title{
A Retrospective Study of 32 Catatonic Patients: Analysis of Clinical, Therapeutic and Evolutional Aspects
}

\section{Chaabouni A, Ellouze F, Ben Soussia R, Fathallah S, Zarrouk L* and Nasr M}

Department of Psychiatry, Hospital Tahar Sfar, University of Monastir, Mahdia, Tunisia

${ }^{*}$ Corresponding author: Zarrouk Lazhar, Department of Psychiatry, Hospital Tahar Sfar, University of Monastir, Mahdia, Tunisia, Tel: 21622676114; E-mail: zarrouk_lazhar@yahoo.fr

Received date: July 22, 2016; Accepted date: August 11, 2016; Published date: August 18, 2016

Copyright: (c) 2016 Chaabouni A, et al. This is an open-access article distributed under the terms of the Creative Commons Attribution License, which permits unrestricted use, distribution, and reproduction in any medium, provided the original author and source are credited.

Citation: Chaabouni A, Ellouze F, Ben Soussia R, et al. A Retrospective Study of 32 Catatonic Patients: Analysis of Clinical, Therapeutic and Evolutional Aspects. Med Clin Rev. 2016, 2:3.

\section{Abstract}

Objective: To analyze the prevalence, the clinical, therapeutic and evolutional aspects of patients treated for catatonia with the intention to improve daily clinical care.

Method: A retrospective study.

Results: Between 2004 and 2013, 32 patients were clinically diagnosed as having catatonia, with a prevalence of $0.97 \%$ among hospitalized patients. The patients were predominantly under 40 years old $(65 \%)$, women $(62 \%)$, single (50\%), jobless (56\%), and of primary education level $(65 \%)$. Most of the patients give a somatic $(31 \%)$ and psychiatric history (59\%). Psychiatric disorders were frequently associated such as schizophrenia $(n=8)$, and mood disorders $(n=4)$, anxiety disorder $(n=2)$, catatonia $(n=1)$ and other psychiatric disorders. Progressive onset (pseudo-neurotic, behavior disorder, activity declining or behavior's modification) was observed in 17 patients. Most clinical events were motor immobility, stupor and mutism. Of the 32 patients, two-thirds had partial response to treatment while complete remission was observed in $16 \%$ of cases. Early somatic complications were frequent $(37.5 \%)$ and two patients died.

Conclusions: A retrospective evaluation of 32 catatonic patients revealed a great variety of clinical events, and psychiatric disorders associated with catatonia, from which schizophrenia and mood disorders are the most frequent. Catatonia is a serious affection considered as an emergency, which remains under diagnosed. So, developing a clinical protocol to improve daily care may be very interesting and complex.

Keywords: Catatonia; Emergency; Transnosographic syndrome; Case series; Complications; Abnormalities

\section{Introduction}

Catatonia is a transnosographic syndrome that stands as a medical emergency characterized by specific motor, mental, vegetative and behavioral abnormalities. It is closely associated with mood, psychotic and somatic disorders as well as intoxicated state $[1,2]$.

Patients with catatonia may experience either an extreme loss of motor skills or a constant hyperactive motor activity. Catatonic patients are at risk of severe complications such as infections, decubitus ulcers, malnutrition, thrombosis and contractures [1]. They can also show autonomic instability, and high percentage of these patients may die in the course of the disease.

The initial treatment is aimed at providing a symptomatic relief. Benzodiazepines (BZD) are the first lines of treatment, and high doses are often required. Electroconvulsivotherapy (ECT) is also an effective treatment option for catatonia [2].

Time delay between first catatonic symptoms and effective treatment is relatively long. This is essentially due to delayed diagnosis [3]. In order to improve clinical care of patients, we aim at evaluating our efforts regularly as well as the efficiency of the clinical care provided. Besides, we try to keep our diagnostic and treatment protocol updated.

In this retrospective study, clinical data are described and our daily practice and development of our clinical workup protocol are discussed.

\section{Methods}

From 2004 to 2013, we examined retrospectively all the medical records of patients hospitalized at the Department of Psychiatry of the University Hospital of Mahdia, Tunisia. Patients diagnosed with catatonia, aged $18-65$ years old were selected. Catatonia diagnosis was established according to criteria of the Diagnostic and Statistical Manual of Mental Disorders Text fourth revision [4].

Catatonic patients were scrutinized by the following variables using a 38 item-questionnaire designed for the study. 


\section{Patient sociodemographic characteristics}

Different characteristics were evaluated: Age, gender marital status, number of children in care, educational level, origin, residence, professional activities, socioeconomic level and social security.

\section{Patients' history}

This involved family history, personal, medical, surgical and psychiatric history, patients' age at the onset of the psychiatric disorders, previous hospitalizations, or catatonic episodes, suicidal and addictive behavior, and forensic act.

\section{Clinical characteristics of a catatonic episode}

The type of onset, clinical events, co morbidities and psychiatric history were evaluated.

\section{Clinical care provided}

Hospitalization mode, length of hospital stay, resort to physical restraint, first line treatment and process at the output were the different clinical features evaluated.

\section{Evolutional aspects}

Responses to treatment, duration of acute catatonic symptoms, longitudinal changes, somatic complications, different complications and risk of mortality in the acute phase were all evaluated.

Data is presented as numbers and percentages means and standard deviation (D.S) or medians and interquartile range, when appropriate, using SPSS for Windows (version 20.0).

\section{Results}

\section{Patients' sociodemographic characteristics}

Between 2004 and 2013, a total of 3304 patients were hospitalized in the Department of Psychiatry. Of these, 32 $(0.97 \%)$ hospitalized patients, aged $18-62$ years old were diagnosed with catatonia. Patients under 40 years old were the most affected, and the age range of the total group was $35.533 \pm 11$ years. Of the 32 patients, $62.5 \%$ were women.

The sex ratio was 0.6 . Sixteen patients were single, while 12 were married, and most of the patients (59\%) did not have any children in care. About two thirds of patients (65.6\%) had a primary educational level. Besides, more than half did not have any professional activities and were from a middle socioeconomic level.

\section{Patients' history}

A summary of patients' history is also shown in Table 1.

The most frequent family history included schizophrenia and mood disorders which accounted for $18 \%$ and $19 \%$ of cases respectively.
Table 1: A summary of the patients' characteristics (from 32 of sample).

\begin{tabular}{|c|c|}
\hline Age, mean \pm S.D. (years) & $35.33 \pm 11$ \\
\hline \multicolumn{2}{|l|}{ Sex } \\
\hline Female & $20(62.5)$ \\
\hline Male & $12(37.5)$ \\
\hline \multicolumn{2}{|l|}{ Marital status, n (\%) } \\
\hline Married & $12(37.5)$ \\
\hline Single & $16(50)$ \\
\hline Divorced & $3(9.4)$ \\
\hline Separated & $1(3.1)$ \\
\hline \multicolumn{2}{|l|}{ Children in care, $\mathrm{n}(\%)$} \\
\hline No children in care & $19(59.4)$ \\
\hline One child & $1(2.1)$ \\
\hline Two children & $5(15.6)$ \\
\hline \multicolumn{2}{|l|}{ Origin, n (\%) } \\
\hline Mahdia & $20(63)$ \\
\hline Sidi Bouzid & - \\
\hline Other & $6(19)$ \\
\hline \multicolumn{2}{|l|}{ Residence, n (\%) } \\
\hline Rural & $17(53.1)$ \\
\hline Urban & $15(46.9)$ \\
\hline \multicolumn{2}{|l|}{ Educational level, n (\%) } \\
\hline Primary & $21(65.6)$ \\
\hline Other & $11(34.4)$ \\
\hline \multicolumn{2}{|l|}{ Professional activities, n (\%) } \\
\hline Jobless & $18(56)$ \\
\hline Worker & $5(15.6)$ \\
\hline Social security, n (\%) & $21(65.6)$ \\
\hline \multicolumn{2}{|l|}{ Family psychiatric history, n (\%) } \\
\hline Schizophrenia & $5(18)$ \\
\hline Mood disorders & $6(19)$ \\
\hline Personal medical and surgical history, n (\%) & $10(31)$ \\
\hline Psychiatric history, n (\%) & $19(59)$ \\
\hline Schizophrenia & $8(25)$ \\
\hline Mood disorders & $4(13)$ \\
\hline Anxiety disorders & $2(6)$ \\
\hline Catatonia & $1(3)$ \\
\hline Other psychiatric history (personality disorder, ...) & $4(12)$ \\
\hline
\end{tabular}


About one third of patients had a medical and surgical history, three patients suffered from epilepsy, and five others respectively had an orbital tumor, hypothyroidism, serious hepatic steatosis, allergic eczema and serious hypokalemia. Besides, a psychiatric history was almost present $159 \%$ of cases) with: Schizophrenia in $25 \%$, mood disorders in $13 \%$, anxiety disorders in $6 \%$ and catatonia in $3 \%$ respectively.

The mean age onset of psychiatric disorders was $27.53 \pm 9$ years. Duration of the psychiatric disorders was 10 years. Furthermore, more than half of the patients had previously required at least one hospitalization in a psychiatry unit, and $40.6 \%$ presented with a previous catatonic episode.

Otherwise, one quarter of catatonic patients had a suicidal behavior, and half of them had an addictive behavior.

\section{Clinical characteristics of a catatonic episode}

The most frequent clinical manifestations were immobility traction, stupor and mutism. Eighteen patients had an isolated catatonic episode. The etiologies included schizophrenia in half of the cases, the major depressive disorder single episode in $18.8 \%$ and scour in $9.4 \%$ of the cases. A histrionic personality was found in $9.4 \%$ of patients. Most of catatonic patients had associated psychosocial and environmental problems.

\section{Clinical care provided}

Concerning consent to hospitalization, the latter was under the free mode in $68.8 \%$ of the cases and the average stay duration was 28 days. Resort to physical restraint was necessary only in a quarter of the cases. Therapeutic control with acute phase comprised BZD for half of the patients and ECT for only $3.6 \%$ of the catatonic patients. Antidepressants and the antipsychotic drugs were prescribed after catatonic resolution.

\section{Evolutional aspects}

Evolution was followed by the early somatic complications in $37.5 \%$ of patients and repetitions of catatonia in $21.9 \%$ of cases. Chronicity and relapse were observed in $15.6 \%$ and $12.5 \%$ of patients respectively. A summary of somatic complications is shown in Table 2.

Table 2: Somatic complications during catatonia progression.

\begin{tabular}{|l|l|}
\hline Type of complications & $\mathbf{n}(\%)$ \\
\hline Metabolic & $12(37.5)$ \\
\hline Infectious & $10(31.2)$ \\
\hline Neurological & $6(19)$ \\
\hline Genito-uninary & $2(6)$ \\
\hline
\end{tabular}

Of the 32 patients, two-thirds had a partial response to treatment while complete remission was observed in $16 \%$ of cases. Mortality at the acute phase was observed in $6.25 \%$ of catatonic patients.

\section{Discussion}

The retrospective evaluation method adopted in the current study is a major drawback. However, the study has shown much important information about catatonia.

This retrospective evaluation of 32 patients revealed different epidemiological, semiological and etiological profiles. Catatonia is a rare emergency which has been overlooked for many decades, but since 1970, it has been increasingly recognized $[5,6]$. The prevalence of catatonia decreases in retrospective studies and increases in prospective ones. This is the case with the fourteen-month prospective study (Abrams and Taylor) which showed 9\% prevalence [7].

In the present study, the mean age of patients was 35.5, which is in accordance with the results found by Krishna [8] and Peralta [9], with extremes going from 19 to 52 years old. The female predominance was also described by England et al. [10].

The psychiatric family history represents an important risk factor. A family history should be taken into account in all cases of rare autosomal-dominant catatonia [11]. Most studies revealed a heavy medical and surgical history including cases such as endocarditis, tuberculosis, cerebral tumor and polytraumatisms $[3,12]$.

Regarding to the psychiatric history, the literature insists that mood disorders are the first etiology of catatonia, about ten times more than schizophrenia $[13,14]$. The present consensus considers that schizophrenia is the second etiology of catatonia. In our study, etiologies were dominated by schizophrenia (half of the cases), since it is the most frequent consultation and hospitalization motive. Also, more than $40 \%$ patients presented with a previous catatonic episode that it may be either a maniac/melancholic relapse or a psychotic one.

While catatonia is only identified as a symptom of schizophrenia in present psychiatric classifications, the current Diagnostic and Statistical Manual of Mental Disorders published by the American Psychiatric Association (DSM 5) [15] did not consider catatonia as a separate disorder, but as associated with certain psychiatric disorders such as bipolar disorder, depression, schizophrenia, post-traumatic stress disorder, drug abuse or over dose [5].

Catatonia may also be seen in many medical disorders such as focal neurological lesions, metabolic disturbances, alcohol withdrawal and abrupt or BZD withdrawal. It can also be an adverse reaction to a prescribed medication such as antipsychotics which may result in neuroleptic malignant syndrome $[5,16,17]$. Autism and mental retardation represent a rare etiology of catatonia.

Different studies $[8,18,19]$ have shown that the most frequent symptoms were stupor, mutism, negativism and cataplexy. Therefore, the diagnosis of catatonia should be made clinically by experienced psychiatrists who have all the available information including patients' history, standard diagnostic instruments and catatonia rating scales [20] in order 
to decrease the time lag between first symptoms and specific first line treatment. Clinical knowledge and experience may shorten the time lag. Furthermore, there is a pressing need to record the precise history of medications and intoxications $[3,21]$

When catatonia is diagnosed, all neuroleptics and psychiatric medications should be avoided except BZD. A high percentage of catatonia was found to be related to somatic disorders, and thus urgent medical examination should be considered. Signs of autonomic dysregulation should be assessed carefully.

In our clinical diagnostic protocol, special attention was given to possible neurological etiology for catatonia as three patients were suffering from epilepsy and another one had an orbital tumor. An overlap between catatonia and epilepsy was already suggested in the original study of Kahlbaum [22].

Concerning treatment, lorazepam is recommended as a first line treatment. Lorazepam should be given intravenously immediately after the diagnosis of catatonia $[23,24]$. ECT is considered as a second-line treatment in all catatonic patients [25-27].

\section{Conclusion}

Catatonia is a rare medical emergency with extreme serious complications that remains underdiagnosed. Early catatonia diagnosis is necessary for a successful therapeutic strategy. During the acute phase, management is primarily based on a symptomatic treatment containing benzodiazepines and/or electroconvulsivotherapy. After resolution, an etiological treatment is sought.

\section{Conflict of Interests}

The authors declare that there is no conflict of interests regarding the publication of this article.

\section{References}

1. Fink M, Taylor MA (2001) The many varieties of catatonia. Eur Arch Psychiatry Clin Neurosci 251: 13-18.

2. Fink M, Taylor AM (2009) The catatonia syndrome. Arch Gen Psychiatry 11: 1173-1177.

3. Tuerlings Joep HAM, van Waaarde JA, Verwey B (2010) A retrospective study of 34 catatonic patients: Analysis of clinical care and treatment. General Hospital Psychiatry 32: 631-635.

4. American Psychiatric Association (2000) Diagnostic and Statistical Manual of Mental Disorders. Text Revision-fourth. Washington DC.

5. Tandon R, Heckers S, Bustillo J (2013) Catatonia in DSM-5. Schizophrenia Res 150: 26-30.

6. Padhy SK, Parakh P, Sridhar M (2014) The catatonia conundrum: controversies and contradictions. Asian J Psychiatry 7: 6-9.

7. Taylor MA, Abrams R (1977) Catatonia: Prevalence and importance in the manie phase of manie depressive illness. Arch General Psychiatry 34: 1223-1225.
8. Krishna KR, Maniar RC, Harbishettar VS (2011) A comparative study of "Idiopathic catatonia" with catatonia in schizophrenia. Asian J Psychiatry 4: 129-33.

9. Peralta V, Campos MS, de Jalon EG, Cuesta MJ (2010) Catatonia signs and criteria in first episode, drug nave, psychotic patients: Psychometric validity and response to antipsychotic medication. Schizophrenia Res 118: 168-175.

10. England ML, Ongur D, Konopaske GT, Karmacharya R (2011) Catatonia in Psychotic Patients: Clinical Features and Treatments Response. J Neuropsychiatry Clin Neurosci 23: 223-226.

11. Northoff G (2002) Catatonia and neuroleptic malignant syndrome: psychopathology and pathophysiology. J Neural Transm 109: 1453-1467.

12. Cottencin O, Thomas P (2007) Catatonia and consultationliaison Psychiatry study on 12 cases. Prog New psychopharmachol Biol Psychiatry 31: 1170-1176.

13. Pommepuy N, Jannuel DLA (2002) Catatonie: Résurgence d'un concept. L'Encéphale. XXVIII Cahier 1: 481-492.

14. Caroff SN, Mann SC, Campbell BC, Sullivan KA (2004) Epidemiology. In: Caroff SN, Mann SC, Francis A, Fricchione GL (eds.) Catatonia: from psychopathology to neurobiology. Washington, DC: APA.

15. American Psychiatric Association (2013) Diagnostic and Statistical Manual of Mental Disorders (5th edn.) Arlington.

16. Rosebush PI, Mazurek MF (1996) Catatonia after benzodiazepine withdrawal. J Clin Psychopharmacology 16: 315-319.

17. Deuschle M, Lederbogen $F$ (2001) Benzodiazepine withdrawalinduced catatonia. Pharmacopsychiatry 34: 41-42.

18. Rosebush P, Hilder Brand AM, Furlon GB (1990) Catatonic Syndrome in a General Psychiatry population. J Clin Psychiatry 51: 357-336

19. Ungvari GS (1994) Leonhard's classification of schizophrenia. A plea for attention. Schizophr Res 12: 269-270.

20. Sienaert P, Rooseleer J, De Fruyt J (2011) Measuring catatonia: A systematic review of rating scales. J Affective Disord 135: 1-9.

21. Fink M, Taylor AM (1890) Catatonia. A clinical's guide to diagnosis and treatment. Cambridge: Cambridge University Press.

22. Kahlbaum KL, Katatonia (1890) Brain, pp: 191-232.

23. Narayanaswamy JC, Tibrewal P, Zutshi A (2012) Clinical predictors of response to treatment in catatonia. General Hospital Psychiatry 34: 312-316.

24. Lin CC, Huang TL (2013) Lorazepam-diazepam protocol for catatonia in schizophrenia: a 21-case analysis. Comprehensive Psychiatry 54: 1210-1214.

25. American Psychiatric Association (2001) The practice of electroconvulsive therapy. American Psychiatric Association (2nd edn.) Washington, D.C.

26. Pompili M, Lester D, Dominici G (2013) Indications for electroconvulsive treatment in schizophrenia: a systematic review. Schizophrenia Res 146: 1-9.

27. Wachtel LE, Hermida A, Dhossche DM (2010) Maintenance electroconvulsive therapy in autistic catatonia: a case series review. Prog Neuropsychopharmacol Biol Psychiatry 34: 581-587. 